\title{
Goniometria da articulação tíbio-tarsal após imobilização temporária com fixador esquelético externo em cães
}

\author{
Tarsocrural joint goniometry after temporary immobilization with external skeletal fixation in dogs
}

\author{
Marcelo Meller Alievi $^{1}$ João Eduardo Schossler ${ }^{2}$ Marcelo Weinsten Teixeira ${ }^{3}$
}

\section{RESUMO}

Neste experimento, foram utilizados 10 cães clinicamente sadios, submetidos à imobilização temporária das articulações tíbio-tarsal, intertarsal e tarso-metatarsiana direita com fixador esquelético externo, tendo por objetivo avaliar através da mensuração da flexão, da extensão e da amplitude articular os danos provocados por esta imobilização. $O$ aparelho de fixação esquelética externa manteve a articulação em um ângulo aproximado de $135^{\circ} \mathrm{e}$ permaneceu por 45 dias. Os animais apresentaram desconforto à manipulação articular para a realização do exame goniométrico no dia da retirada do aparelho, demonstrando dor na flexão e na extensão passiva do membro imobilizado, sendo que tal dificuldade desapareceu após três dias. Foi observado que a imobilização articular temporária obtida pela fixação esquelética externa promove perda estatisticamente significativa nos graus de flexão, de extensão $e$ de amplitude articular tíbio-tarsal imediatamente após a remoção do fixador externo. Porém, após sete dias desta remoção para extensão e quinze dias para flexão e amplitude articular, esta perda não é mais verificada.

Palavras-chave: jarrete, ortopedia, articular, cão.

\section{ABSTRACT}

In this study, 10 clinically healthy adult mongrel dogs were submitted to the temporary immobilization of the hock with external skeletal fixation. The aim of this procedure was to evaluate, using a manual goniometer, the flexion, the extension and the range of motion of the tarsocrural joint after immobilization in a functional angle of $135^{\circ}$ for 45 days. The animals presented discomfort in relation to the articular manipulation for the goniometric exam on the day of the removal of the apparatus, demonstrating pain in the passive flexion and extension of the limb, and such difficulty disappeared after three days. It was observed that the temporary immobilization of the articulation obtained by the external skeletal fixation promotes statistically significant loss in the degree of flexion, extension and range of motion of the tarsocrural joint immediately after the removal of the apparatus. However, after seven days of this removal, for extension, and fifteen days, for flexion and range of motion of the articulation, this loss is not verified.

Key words: hock, orthopaedics, articular, dog.

\section{INTRODUÇÃO}

Os primeiros relatos da utilização do aparelho de fixação esquelética externa em humanos datam do ano de 1897, sendo Ehmer o primeiro a adaptar a técnica para a veterinária e criar o aparelho conhecido até hoje como Kirschner-Ehmer (EGGER, 1991).

Uma vantagem adicional do método de fixação esquelética externa é a possibilidade de imobilizar articulações temporariamente, sendo utilizada principalmente nos casos de fraturas com fragmentos distais muito pequenos (BEHRENS et al., 1983), nas lesões abrasivas que envolvem a região articular (DIAMOND et al., 1999) e na substituição ou síntese de ligamentos e tendões rompidos (MORSHEAD \& LEEDS, 1984; ARON, 1987).

MORSHEAD \& LEEDS (1984) e TOOMBS et al. (1989) concluíram que a imobilização articular temporária utilizando fixador esquelético externo é bem tolerada pelos animais, permitindo retorno precoce à utilização funcional do membro. Porém, BJORLING \& TOOMBS (1982) citaram que a imobilização articular prolongada pode levar à atrofia muscular e diminuição na amplitude dos movimentos da articulação. EVANS et al. (1960) verificaram em ratos que a contratura tanto dos músculos como da cápsula articular, a proliferação de tecido conectivo intracapsular e a formação de

${ }^{1}$ Médico Veterinário, Doutorando do Programa de Pós-graduação em Medicina Veterinária, área de Cirurgia, Universidade Federal de Santa Maria (UFSM), Santa Maria, RS.

${ }^{2}$ Médico Veterinário, Doutor, Professor Adjunto, Departamento de Clínica de Pequenos Animais, Centro de Ciências Rurais, UFSM, 97105-900, Santa Maria, RS. Autor para correspondência. E-mail: schossle@lince.hcv.ufsm.br

${ }^{3}$ Médico Veterinário, Mestre, Professor Assistente, Departamento de Morfologia, Universidade Federal Rural de Pernambuco. 
aderências são os responsáveis pela restrição da movimentação articular. Além disso, a imobilização prolongada de uma articulação pode levar à osteoporose por desuso (UHTHOFF \& JAWORSKI, 1978), à diminuição acentuada de proteoglicanos da cartilagem articular (BEHRENS et al., 1989) e ao desenvolvimento de osteoartrite (LANGENSKIÖLD et al., 1979).

Normalmente a imobilização da articulação tíbio-tarsal utilizando-se a fixação esquelética externa deve ser feita mantendo-se um ângulo aproximado de 130 a 150 graus. Esta variação ocorre devido à diferença na conformação corporal entre as raças e ao tipo e local do traumatismo. A utilização de um goniômetro é recomendada para a mensuração do ângulo normal do membro contralateral, não traumatizado (TOOMBS et al., 1989).

A mensuração goniométrica da flexão e da extensão de uma articulação é uma técnica simples e que fornece informações adicionais aos clínicos ou aos pesquisadores quanto ao grau de alteração articular e quanto à evolução pós-operatória de pacientes ortopédicos. Os valores médios de extensão, de flexão e de amplitude articular tíbio-tarsal em cães hígidos são, respectivamente, 175, 40 e 134 graus (MANN et al., 1988).

TEIXEIRA (1998), em um estudo utilizando a fixação esquelética externa para imobilizar as articulações tíbio-tarsal, intertarsal e tarsometatarsiana como forma de tratamento para fraturas distais de tíbia em cães, observou que, após a retirada do aparelho, alguns animais, apesar de voltarem a caminhar com pouca ou nenhuma claudicação, apresentaram diminuição no grau de flexão e de extensão da articulação tíbio-tarsal. O referido autor comentou que esta complicação poderia ser conseqüência do método de fixação utilizado ou seqüela da lesão óssea e de tecidos moles ocorrida no momento do traumatismo. Tal fato motivou a realização do presente trabalho, permitindo assim avaliar isoladamente, através da mensuração da flexão, da extensão e da amplitude articular, os danos provocados pela utilização da fixação esquelética externa como método de imobilização temporária da articulação tíbiotarsal.

\section{MATERIAL E MÉTODOS}

Foram utilizados 10 cães clinicamente sadios, de ambos os sexos, sem raça definida, com idade variando entre oito meses e seis anos, peso corpóreo de 10 a $20 \mathrm{~kg}$, provenientes do Biotério Central da UFSM. Os animais foram inicialmente submetidos à mensuração da flexão e da extensão da articulação tíbio-tarsal bilateral, utilizando-se um goniômetro manual ${ }^{a}$ (Figura 1). Através da subtração do valor da extensão pelo da flexão, foi obtida a amplitude articular.

Após jejum de 12 horas e tricotomia que envolveu a região da articulação do joelho até a articulação metatarso-falangeana direita, cada cão recebeu como medicação pré-anestésica, acepromazina $^{\mathrm{b}}$, na dose de $0,2 \mathrm{mgkg}^{-1}$, associada a fentanil ${ }^{\mathrm{c}}$, na dose de $0,005 \mathrm{mgkg}^{-1}$, por via intramuscular. Após 15 minutos, a indução anestésica foi realizada com $10 \mathrm{mgkg}^{-1}$ de tiopental sódico ${ }^{\mathrm{d}}$, por via intravenosa, e a manutenção com halotano ${ }^{\mathrm{e}}$ e oxigênio em circuito anestésico semi-fechado. A anti-sepsia da área operatória foi efetuada com o esquema álcooliodo-álcool e após esta foi delimitada por panos de campo esterilizados. Em seguida, foi confeccionado o aparelho de fixação esquelética externa para imobilizar as articulações tíbio-tarsal, intertarsal e tarsometatarsiana direita.

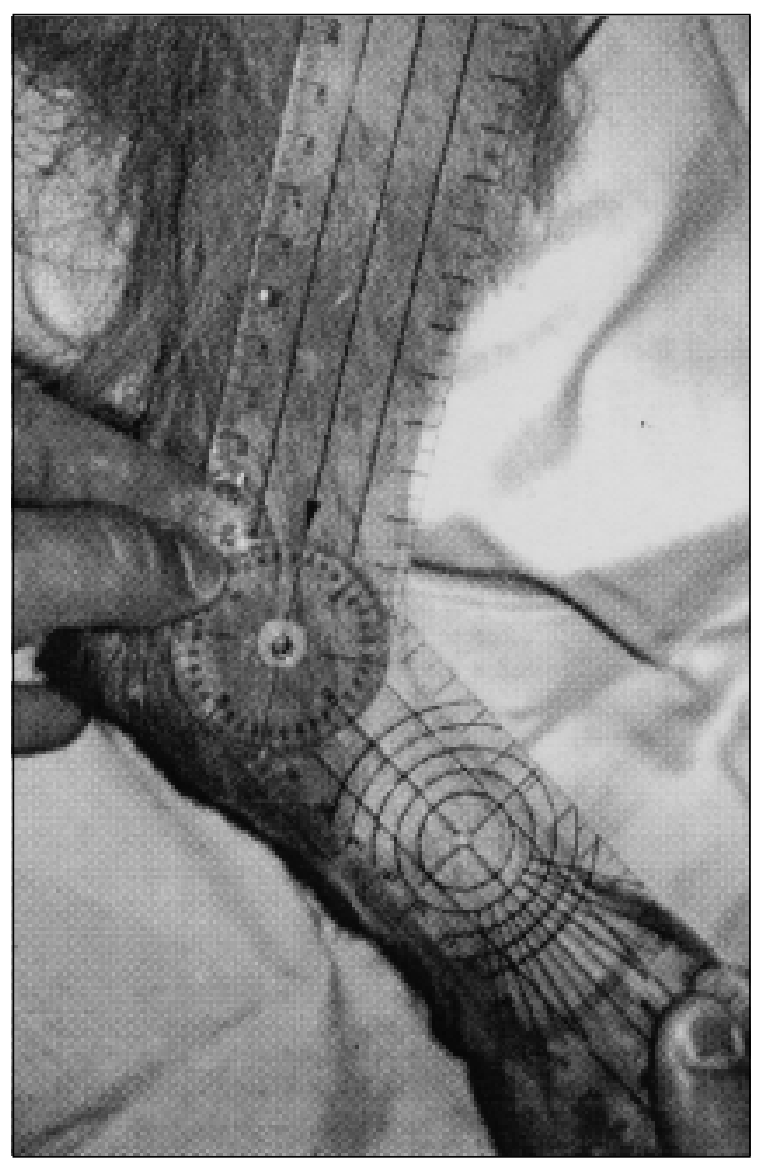

Figura 1 - Avaliação da amplitude articular tíbio-tarsal de um cão utilizando goniômetro manual. 
A fixação externa constou da inserção, por via percutânea, de cinco pinos de Steinmann com diâmetro de aproximadamente $20 \%$ do diâmetro ósseo. Dois pinos foram inseridos na região diafisária distal da tíbia, um no calcâneo e dois nos metatarsianos. Os pinos formavam um ângulo de aproximadamente 70 graus em relação ao eixo longitudinal dos ossos, e, após a sua introdução, os mesmos foram envergados de maneira a ficarem paralelos ao eixo longitudinal dos ossos, mantendo uma distância da pele de aproximadamente $3 \mathrm{~cm}$.

Após tais procedimentos, a articulação tíbio-tarsal foi mantida em um ângulo aproximado de 135 graus. Os pinos foram unidos utilizando-se acrílico autopolimerizável ${ }^{\mathrm{f}}$ como haste de fixação externa, uma na face lateral e outra na medial do membro.

Foi utilizado flunixin meglumine ${ }^{\mathrm{g}}$ como agente antiinflamatório e analgésico, na dose diária de $1 \mathrm{mgkg}^{-1}$, durante os três primeiros dias de pósoperatório. Os animais permaneceram com o aparelho de fixação esquelética externa por 45 dias, sendo realizada durante este período, limpeza cutânea no local de inserção dos pinos, com solução fisiológica e gaze. Após a remoção do fixador externo, foram avaliadas diariamente com goniômetro a flexão, a extensão e a amplitude articular tíbio-tarsal. Esse procedimento foi efetuado por 15 dias, tendo como valores de referência os da avaliação pré-operatória.

Para a análise estatística, foram realizados Análise da Variância, Teste F e Teste de Tukey, sendo utilizado o pacote estatístico SAS for Windows, versão 6.11, e o nível de significância de 0,05 .

\section{RESULTADOS E DISCUSSÃO}

Os dados referentes às médias e aos desvios padrão da flexão, da extensão e da amplitude articular nos diferentes períodos de observação estão contidos na tabela 1 .
Assim como o recomendado por TOOMBS et al. (1989), procurou-se imobilizar a articulação tíbiotarsal em um ângulo aproximado de 135 graus. A manutenção de tal angulação permitiu que os animais apoiassem adequadamente o membro durante a permanência do aparelho de fixação esquelética externa. Cabe salientar que pode haver variações quanto ao valor desse ângulo, sendo maior em raças como o Chow-Chow, que mantém essa articulação pouco flexionada, e menor em outras, como o Pastor Alemão, que a mantém bastante flexionada (MANN et al., 1988). Com isso, é recomendada a mensuração do ângulo normal no membro contralateral, não traumatizado (TOOMBS et al., 1989).

Apesar de DIAMOND et al. (1999) citarem a ocorrência de complicações como quebra da barra de conexão ou remoção prematura do aparelho pelo animal quando se utiliza fixação esquelética externa para promover imobilização articular temporária, no presente estudo, não foram observadas quaisquer alterações no fixador, estando firme e intacto no dia da sua retirada. Provavelmente as complicações encontradas por DIAMOND et al. (1999) possam ser atribuídas à utilização de fixador esquelético externo tipo I ou unilateral, enquanto no presente estudo foi utilizado fixador tipo II ou bilateral, sendo o segundo consideravelmente mais resistente que o primeiro.

Após a remoção do fixador, foi observado que os animais tiveram retorno quase que imediato ao uso funcional do membro, exceto um, que apresentou processo inflamatório acentuado e anquilose da articulação tíbio-tarsal, com perda da mobilidade e utilização parcial do membro, mesmo 15 dias após a retirada do aparelho de fixação esquelética externa. Neste animal, iniciou-se fisioterapia com movimentos passivos de flexão e extensão três vezes ao dia durante 30 dias, sem sucesso. Segundo DIAMOND et al. (1999) tal complicação pode ter sido provocada pela ocorrência de fibrose periarticular e pelo desenvolvimento de alterações degenerativas

Tabela 1 - Médias e desvios padrão da flexão, da extensão e da amplitude articular, em graus, obtida por exames goniométricos no período pré-operatório, imediato, sete e quinze dias após a remoção do fixador esquelético externo, que imobilizou as articulações tíbiotarsal, intertarsal e tarso-metatarsiana por 45 dias*.

\begin{tabular}{lcccc}
\hline Períodos & & Flexão & Extensão & Amplitude articular \\
\hline Pré-operatório & & $56,55 \pm 1,66^{\mathrm{a}}$ & $158,22 \pm 2,68^{\mathrm{a}}$ & $101,66 \pm 3,87^{\mathrm{a}}$ \\
& Imediato & $88,33 \pm 2,91^{\mathrm{c}}$ & $134,66 \pm 6,40^{\mathrm{b}}$ & $46,33 \pm 8,74^{\mathrm{c}}$ \\
Pós-remoção & 7 dias & $68,55 \pm 3,64^{\mathrm{b}}$ & $150,88 \pm 4,04^{\mathrm{a}}$ & $82,33 \pm 5,85^{\mathrm{b}}$ \\
& 15 dias & $57,66 \pm 1,50^{\mathrm{a}}$ & $156,66 \pm 2,82^{\mathrm{a}}$ & $99,00 \pm 3,64^{\mathrm{a}}$ \\
\hline
\end{tabular}

a, b, c - Médias seguidas das mesmas letras, na mesma coluna, não diferem entre si pelo teste de Tukey $(\mathrm{P}<0,05)$.

* - O animal que apresentou anquilose foi excluído da análise estatística. 
próximas à articulação, oriundas do processo inflamatório acentuado que o cão apresentou. Para fins estatísticos, o animal não foi incluído na análise. Assim como o relatado por MANN et al. (1988), os animais apresentaram no dia da retirada do aparelho resistência à manipulação articular para a realização do exame goniométrico, demonstrando dor na flexão e na extensão passivas do membro imobilizado. Este fato é justificado pelo tempo de imobilização articular, que está associado à atrofia muscular (BJORLING \& TOOMBS, 1982), contratura da musculatura e da cápsula articular, proliferação de tecido conectivo intracapsular e formação de aderência (EVANS et al., 1960). A dificuldade em permitir o exame desapareceu no terceiro dia após a retirada do aparelho.

Os valores de flexão e extensão iniciais encontrados nas articulações tíbio-tarsais no presente estudo foram menores do que os encontrados por MANN et al. (1988), podendo esta diferença ser explicada pelo fato de que esses autores, para a realização das mensurações, submeteram os cães à anestesia geral inalatória, o que certamente promoveu maior relaxamento muscular e maior facilidade na realização do exame, com isso aumentando a amplitude de movimentação articular. No presente experimento, o movimento articular foi passivo, ou seja, o limite máximo de movimentação foi aquele permitido pelo animal, enquanto no estudo realizado por MANN et al. (1988) a mensuração foi efetuada com amplitude articular máxima, sem interferência do animal.

\section{CONCLUSÃO}

Com os resultados obtidos neste trabalho conclui-se que a imobilização temporária da articulação tíbio-tarsal promovida pela fixação esquelética externa promove perda estatisticamente significativa na flexão, na extensão e na amplitude articular imediatamente após a remoção do fixador, porém, esta perda não é mais verificada após sete dias para extensão e quinze dias para flexão e amplitude articular.

\section{FONTES DE AQUISIÇÃO}

a- Goniômetro: Carci Indústria e Comércio de aparelhos cirúrgicos e ortopédicos LTDA, São Paulo, SP.

b- Acepran: Univet S/A Indústria Veterinária, São Paulo, SP. c- Fentanil: Cristália Produtos Químicos e Farmacêuticos Ltda, Itapira, SP.

d- Thionembutal: Abbott Laboratórios do Brasil Ltda, São Paulo, SP.

e- Halotano: Hoechst do Brasil, Suzano, SP. f- Jet Acrílico: Artigos Odontológicos Clássico, São Paulo, SP. g- Banamine: Schering-Plough Veterinária, Rio de Janeiro, RJ.

\section{REFERÊNCIAS BIBLIOGRÁFICAS}

ARON, D.N. Prosthetic ligament replacement for severe tarsocrural joint instability. Journal of the American Animal Hospital Association, v.23, n.1, p.41-55, 1987.

BEHRENS, F. et al. Unilateral external fixation for severe open tibial fractures - Preliminary report of a prospective study. Clinical Orthopaedics and Related Research, n.178, p.111-120, 1983.

BEHRENS, F.; KRAFT, E.L.; OEGEMA, T.R. Biochemical changes in articular cartilage after joint immobilization by casting or external fixation. Journal of Orthopaedic Research, v.7, n.3, p.335-343, 1989.

BJORLING, D.E.; TOOMBS, J.P. Transarticular application of the Kirschner-Ehmer splint. Veterinary Surgery, v.11, n.1, p.34-38, 1982 .

DIAMOND, D.W.; BESSO, J.; BOUDRIEAU, R.J. Evaluation of joint stabilization for treatment of shearing injuries of the tarsus in 20 dogs. Journal of the American Animal Hospital Association, v.35, n.2, p.147-153, 1999.

EGGER, E.L. Complications of external fixation. The Veterinary Clinics of North America: Small Animal Practice, v.21, n.4, p.705-733, 1991.

EVANS, E.B. et al. Experimental immobilization and remobilization of rat knee joints. Journal of Bone and Joint Surgery, v.42A, n.5, p.737-758, 1960.

LANGENSKIÖLD, A.; MICHELSSON, J.E.; VIDEMAN, T. Osteoarthritis of knee in the rabbit produced by immobilization. Acta Orthopaedica Scandinavica, v.50, p.1-14, 1979.

MANN, F.A.; WAGNER-MANN, C.; TANGNER, C.H. Manual goniometric measurement of the canine pelvic limb. Journal of the American Animal Hospital Association, v.24, n.2, p.189-194, 1988.

MORSHEAD, D.; LEEDS, E.B. Kirschner-Ehmer apparatus immobilization following achilles tendon repair in six dogs. Veterinary Surgery, v.13, n.1, p.11-14, 1984.

TEIXEIRA, M.W. Osteossíntese distal de tíbia por transfixação tíbio-tarsal temporária em pequenos animais. 1998. 46f. Dissertação (Mestrado em Medicina Veterinária) - Programa de Pós-graduação em Medicina Veterinária, Universidade Federal de Santa Maria.

TOOMBS, J.P.; ARON, D.N.; BASINGER, R.R. Angled connecting bars for transarticular application of KirschnerEhmer external fixation splints. Journal of the American Animal Hospital Association, v.25, n.2, p.213-216, 1989.

UHTHOFF, H.K.; JAWORSKI, Z.F.G. Bone loss in response to long-term immobilisation. The Journal of Bone and Joint Surgery, v.60B, n.3, p.420-429, 1978. 